\title{
Non-Gaussianity from preheating
}

\author{
Kari Enqvist, ${ }^{1,3}$ Asko Jokinen, ${ }^{2}$ Anupam Mazumdar, ${ }^{2}$ Tuomas Multamäki, ${ }^{2}$ and Antti Väihkönen ${ }^{1,3}$ \\ ${ }^{1}$ Helsinki Institute of Physics, University of Helsinki, P.O. Box 64, FIN-00014 Helsinki, Finland \\ ${ }^{2}$ NORDITA, Blegdamsvej-17, Copenhagen-2100, Denmark \\ ${ }^{3}$ Department of Physical Sciences, University of Helsinki, P.O. Box 64, FIN-00014 Helsinki, Finland \\ (Received 23 November 2004; revised manuscript received 7 February 2005; published 29 April 2005)
}

We consider a two-field model for inflation where the second order metric perturbations can be amplified by a parametric resonance during preheating. We demonstrate that there can arise a considerable enhancement of non-Gaussianity sourced by the local terms generated through the coupled perturbations. We argue that the non-Gaussianity parameter could be as large as $f_{\mathrm{NL}} \approx 50$. Our results may provide a useful test of preheating in future cosmic microwave background experiments.

Introduction. - Preheating, first realized in [1] and worked out in detail in [2-4], may play an important role in understanding the hot thermal Universe after the end of inflation. Preheating can occur during the coherent oscillation of the homogeneous inflaton condensate if a resonant production of particles takes place due to a temporary vacuum instability. During this phase it is also possible to amplify the gravitational fluctuations or the metric fluctuation to the super-Hubble scale [5,6] (for a general first order linear perturbation theory, see [7]). So far the analysis has been limited to the first order metric and matter perturbations, although there have also been some attempts to understand the higher order effects [8]. In some cases the inflaton could also fragment into nontopological solitons, but then the inhomogeneities remain below the subhorizon scale [9].

Since cosmic microwave background (CMB) experiments such as WMAP [10] have now reached a precision whereby cosmological models can be put to the test, preheating may also move from the theoretical playground to observational scrutiny. In this Letter, we propose that nonGaussianities in the CMB fluctuations could provide a useful tool for studying preheating.

The simplest single-field inflationary models produce scale invariant Gaussian fluctuations, assuming that the initial state is the standard vacuum. However, nonGaussianities are expected to be generated at some level, either because of inflaton self-couplings [11], nonstandard initial vacuum state [12], or because of a host of other reasons often involving models beyond the simple singlefield inflation [13-15] (for a review, see [16]). For example, in hybrid-type scenarios with two fields the fluctuations of the "waterfall" field may give rise to large nonGaussianities during inflation [15].

Major non-Gaussianities may also be generated after inflation [17]. Here we focus on non-Gaussianities during the preheating stage, although our approach is quite generic and applicable to any scalar condensate responsible for reheating the Universe, such as a supersymmetric flat direction [18], for which we may employ the formalism developed in [15]. We will consider a simple toy model of two fields, making some well motivated physical assumptions regarding the motion of the coupled fields. Our starting point is an oscillating inflaton coupled to another field, which for simplicity is assumed to have a vanishing vacuum expectation value. Therefore, classically the second field is sitting at the bottom of its potential. However, its fluctuation about the background solution is nonvanishing, giving rise to matter perturbations which naturally are higher order, mainly chi-squared fluctuations [19], which may source the metric perturbations and lead to nonGaussianities. We illustrate that, although the first order metric perturbations do not grow, for long wavelength super-Hubble horizon modes the second order metric fluctuations can grow exponentially, highlighting the potential for a large non-Gaussian signature arising from preheating.

For simplicity, for obtaining the estimates we will neglect the expansion of the Universe. This may seem rather restrictive, but since the homogeneous condensate oscillates coherently with a frequency larger than the Hubble expansion rate during the initial stages of preheating, the only relevant time scale is the mass of the coherently oscillating field. Of course, for detailed numerical values, the expansion as well as backreaction effects will be important.

Basic equations. - Let us consider a two-field model with the potential

$$
V=\frac{1}{2} m_{\varphi}^{2} \varphi^{2}+\frac{1}{2} g^{2} \varphi^{2} \sigma^{2}
$$

where $\varphi$ is the homogeneous scalar condensate coherently oscillating with a mass $m_{\varphi}$. We assume $\varphi$ to be our inflaton. The $\sigma$ field will be created resonantly by virtue of the coupling term.

For simplicity and for the sake of clarity we assume that the vacuum expectation value (VEV) of $\sigma$ vanishes, $\langle\sigma\rangle=$ 0 , which makes it possible to obtain analytic approximations from the second order perturbation equations, as we show below. Such a situation occurs if the $\sigma$ field is driven to the minimum of its potential during inflation. 
The metric in our case is given by

$$
\begin{gathered}
g_{00}=-a(\eta)^{2}\left(1+2 \phi^{(1)}+\phi^{(2)}\right), \quad g_{0 i}=0, \\
g_{i j}=a(\eta)^{2}\left(1-2 \psi^{(1)}-\psi^{(2)}\right) \delta_{i j},
\end{gathered}
$$

where we are using the generalized longitudinal gauge and neglect the vector and tensor perturbations. Here $\eta$ is the conformal time and $a(\eta)$ is the scale factor. We divide the fields into background and perturbations,

$$
\begin{aligned}
& \varphi=\varphi_{0}(\eta)+\delta^{(1)} \varphi(\eta, \mathbf{x})+\frac{1}{2} \delta^{(2)} \varphi(\eta, \mathbf{x}), \\
& \sigma=\delta^{(1)} \sigma(\eta, \mathbf{x})+\frac{1}{2} \delta^{(2)} \sigma(\eta, \mathbf{x}),
\end{aligned}
$$

where the background value for $\sigma$ is assumed to vanish.

The background equations of motion are found to be

$$
\begin{gathered}
3 \mathcal{H}^{2}=\frac{1}{2 M_{p}^{2}} \varphi_{0}^{\prime 2}+\frac{M_{p}^{2}}{2} a^{2} m_{\varphi}^{2} \varphi_{0}^{2}, \\
0=\varphi_{0}^{\prime \prime}+2 \mathcal{H} \varphi_{0}^{\prime}+a^{2} m_{\varphi}^{2} \varphi_{0},
\end{gathered}
$$

while the $\sigma_{0}$ equation is trivial, and $\mathcal{H}$ denotes the Hubble expansion rate in conformal time. We denoted the reduced Planck mass as $M_{p} \sim 2.4 \times 10^{18} \mathrm{GeV}$.

The relevant first order perturbation equations can be written in the form [15]

$$
\begin{aligned}
\phi^{(1)^{\prime \prime}}-\partial_{i} \partial^{i} \phi^{(1)}+2(\mathcal{H}- & \left.\frac{\varphi_{0}^{\prime \prime}}{\varphi_{0}^{\prime}}\right) \phi^{(1)^{\prime}} \\
& +2\left(\mathcal{H}^{\prime}-\frac{\varphi_{0}^{\prime \prime}}{\varphi_{0}^{\prime}} \mathcal{H}\right) \phi^{(1)}=0,
\end{aligned}
$$

$$
\delta^{(1)} \sigma^{\prime \prime}+2 \mathcal{H} \delta^{(1)} \sigma^{\prime}-\partial_{i} \partial^{i} \delta^{(1)} \sigma+g^{2} \varphi_{0}^{2} \delta^{(1)} \sigma=0 .
$$

Note that there are no metric perturbations in Eq. (7). This is due to assuming a vanishing VEV for $\sigma$. If it were nonvanishing, we would not be able to decouple the metric fluctuations from the perturbations in the $\sigma$ field. Now the $\sigma$ part can be solved separately and for the rest the usual one field results apply.

Parametric resonance. - Let us now turn to the parametric resonance for $\delta^{(1)} \sigma_{k}$. Throughout this section we neglect the expansion of the Universe, which results in $a=$ 1 and $\eta=t$, i.e., conformal and cosmic time are equal, since the coherent oscillations begin only when $m_{\varphi} \gg \mathcal{H}$. Hence the excitation of $\sigma_{k}$ will follow the equation of motion valid for a narrow parametric resonance regime $[3,5]$,

$$
\frac{d^{2} \delta^{(1)} \sigma_{k}}{d z^{2}}+\left(A_{k}-2 q \cos 2 z\right) \delta^{(1)} \sigma_{k}=0,
$$

where $A_{k}=k^{2} / m_{\varphi}^{2}+2 q, q=g^{2} \Phi^{2} / 4 m_{\varphi}^{2}$, and $z=m_{\varphi} t$. The amplitude of the oscillations is denoted by $\Phi$. In a narrow resonance regime $q<1$. In this regime the perturbation grows exponentially as $\delta^{(1)} \sigma_{k} \propto \exp \left(\mu_{k} m_{\varphi} t\right)$, where

$$
\mu_{k}=\sqrt{(q / 2)^{2}-\left(2 k / m_{\varphi}-1\right)^{2}} .
$$

Therefore, we have a resonance for the modes $k$ when $k_{-}<k<k_{+}$with $k_{ \pm}=\left(m_{\varphi} / 2\right)(1 \pm q / 2)$.

For our purposes it is sufficient to estimate the solution to be independent of $k$ in the resonance band. Therefore, we estimate $\delta^{(1)} \sigma_{\text {eff }} \equiv A \exp \left(\mu_{\text {eff }} m_{\varphi} t\right)$, where $\mu_{\text {eff }}=$ $\mu_{\max } / 2=q / 4$ in the resonance band and $\delta^{(1)} \sigma_{\text {eff }}=0$ otherwise; here $A$ is an amplitude after the end of inflation.

The amplitude depends on the effective mass of $\sigma$ field. If $g \varphi_{0} \leq m_{\varphi}$, then the initial perturbations are given by $A \sim \delta^{(1)} \sigma_{k} \sim\left(H / \sqrt{2 k^{3}}\right)(k / a H)^{3 / 2-\nu}$, where $\nu=$ $\sqrt{9 / 4-g^{2} \varphi_{0}^{2} / H^{2}}$. For a narrow resonance regime, $g \leq$ $H / \varphi_{0} \sim H / M_{p} \ll 1$, the spectrum follows that of the inflaton, where we assumed at the end of inflation $\varphi_{0} \sim$ $M_{p}$. For a broad resonance regime, $q>1$, and $g \varphi_{0} \gg m_{\varphi}$, there the amplitude of the perturbations for $\sigma$ field will be suppressed compared to that of the inflaton; see [6].

Perturbations. - The first order metric perturbation at the end of inflation for a single field is given by [7],

$$
\phi^{(1)}=\frac{H}{\dot{\varphi}_{0}} \delta^{(1)} \varphi \approx-\frac{\varphi_{0}}{2 M_{p}^{2}} \delta^{(1)} \varphi,
$$

where the quantities on the right-hand side are calculated at the horizon crossing. We obtained the final result by using slow roll equations of motion. Since $g \ll 1$, then during inflation we can effectively treat $\delta^{(1)} \sigma \sim \delta^{(1)} \varphi$. This determines the initial conditions before preheating starts.

In the second order we are only interested in the gravitational perturbation, whose equation can be written in an expanding background as [15]

$$
\phi^{(2) \prime \prime}+2\left(\mathcal{H}-\frac{\varphi_{0}^{\prime \prime}}{\varphi_{0}^{\prime}}\right) \phi^{(2) \prime}+2\left(\mathcal{H}^{\prime}-\frac{\varphi_{0}^{\prime \prime}}{\varphi_{0}^{\prime}} \mathcal{H}\right) \phi^{(2)}-\partial_{i} \partial^{i} \phi^{(2)}=J_{\sigma, \text { local }}+J_{\sigma \text {,non-local }}+J_{\text {rest }},
$$

where the source terms $J$ are quadratic combinations of first order perturbations; in particular,

$$
J_{\sigma, \text { local }}=-\frac{2}{M_{p}^{2}}\left(\delta^{(1)} \sigma^{\prime}\right)^{2}+\frac{a^{2}}{M_{p}^{2}} \frac{\delta^{2} V}{\delta \sigma^{2}}\left(\delta^{(1)} \sigma\right)^{2}
$$

$J_{\sigma \text {,non-local }}$ involves an inverse spatial Laplacian, thus rendering it nonlocal, while $J_{\text {rest }}$ consists of metric and $\varphi$ perturbations. Note that the left-hand side of the above equation is identical to the first order equation, Eq. (6).

Fourier transforming $J_{\sigma \text {,local }} \rightarrow J_{k}$ [with our convention $\left.f(\boldsymbol{x})=(1 / 2 \pi)^{3} \int d^{3} \boldsymbol{k} e^{\boldsymbol{k} \cdot \boldsymbol{x}} f(\boldsymbol{k})\right]$ we end up with the 
convolutions

$$
\begin{aligned}
J_{k}= & -\frac{2}{M_{p}^{2}(2 \pi)^{3}} \int d^{3} \boldsymbol{k}^{\prime} \delta^{(1)} \sigma_{\boldsymbol{k}^{\prime}}^{\prime} \delta^{(1)} \sigma_{\boldsymbol{k}-\boldsymbol{k}^{\prime}}^{\prime} \\
& +\frac{1}{M_{p}^{2}} \frac{\delta^{2} V}{\delta \sigma^{2}} \frac{1}{(2 \pi)^{3}} \int d^{3} \boldsymbol{k}^{\prime} \delta^{(1)} \sigma_{\boldsymbol{k}^{\prime}} \delta^{(1)} \sigma_{\boldsymbol{k}-\boldsymbol{k}^{\prime}}
\end{aligned}
$$

The object then is to compute the convolutions. We are interested in their contributions at large scales and, to that end, we take the limit $k \rightarrow 0$; we also assumed $a=1$. Since the mode function $\delta^{(1)} \sigma_{\text {eff }}$ only depends on the magnitude of the vector $\boldsymbol{k}$, the angular integration can be carried out trivially. The time derivative only produces a constant factor. Thus, we obtain

$$
\begin{aligned}
J_{k}= & -2 \frac{1}{M_{p}^{2}} \frac{4 \pi}{(2 \pi)^{3}} \int d k^{\prime} k^{\prime 2}\left(\delta^{(1)} \sigma_{k^{\prime}}^{\prime}\right)^{2} \\
& +\frac{a^{2}}{M_{p}^{2}} \frac{\delta^{2} V}{\delta \sigma^{2}} \frac{4 \pi}{(2 \pi)^{3}} \int d k^{\prime} k^{\prime 2}\left(\delta^{(1)} \sigma_{k^{\prime}}\right)^{2} \\
= & {\left[-\frac{2 \mu_{\mathrm{eff}}^{2} m_{\varphi}^{2}}{M_{p}^{2}}+\frac{1}{M_{p}^{2}} \frac{\delta^{2} V}{\delta \sigma^{2}}\right] \delta^{(1)} \sigma_{\mathrm{eff}}^{2} \frac{4 \pi}{(2 \pi)^{3}} \int_{k_{-}}^{k_{+}} d k^{\prime} k^{\prime 2}, }
\end{aligned}
$$

where in the last step we have assumed that the $k$ dependence of the amplitude $A$ can be ignored. If we are working in a narrow resonance regime with $q<1$, the integral can be written as

$$
\int_{k_{-}}^{k_{+}} d k^{\prime} k^{2}=\frac{1}{3}\left(\frac{m_{\varphi}}{2}\right)^{3}\left[3 q+2\left(\frac{q}{2}\right)^{3}\right] \simeq q\left(\frac{m_{\varphi}}{2}\right)^{3} .
$$

We can now write the source term as

$$
\begin{aligned}
J_{k} & =\frac{4 \pi}{(2 \pi)^{3}} q\left(\frac{m_{\varphi}}{2}\right)^{3}\left[-\frac{q^{2} m_{\varphi}^{2}}{8 M_{p}^{2}}+\frac{1}{M_{p}^{2}} \frac{\delta^{2} V}{\delta \sigma^{2}}\right] A^{2} e^{q m_{\varphi} t / 2} \\
& =\frac{2 m_{\varphi}^{2} q}{M_{p}^{2}}\left[1-\frac{q}{16}\right] \times B e^{q m_{\varphi} t / 2} .
\end{aligned}
$$

Where $B=\left(q / 8 \pi^{2}\right)\left(m_{\varphi} / 2\right)^{3} A^{2}$. It is worth noting that the source $J_{k}$, which we study at the large scale limit $k \sim 0$, is actually generated by first order local perturbations on much smaller scales $\left(k_{-}<k<k_{+}\right)$.

Non-Gaussianity. - Consider Eq. (11) in $k$ space. The homogeneous part is the same as in the first order. Therefore we know that the homogeneous solutions are well behaved. Barring accidental cancellations we may assume that the local terms we have considered are representative of the exponential behavior of the source; see Eq. (12). There is also a nonexponential part which naturally becomes quickly insignificant. In order to estimate the behavior of $\phi^{(2)}$ at large scales $(k \sim 0)$ we neglect the expansion of the Universe and drop the terms with $\mathcal{H}$. The approximated metric perturbation then reads

$$
\begin{aligned}
\phi_{k}^{(2) \prime \prime}-2 \frac{\varphi_{0}^{\prime \prime}}{\varphi_{0}^{\prime}} \phi_{k}^{(2) \prime}= & \frac{2 m_{\varphi}^{2} q}{M_{p}^{2}}\left[1-\frac{q}{16}\right] \times B e^{q m_{\varphi} t / 2} \\
& +(\text { nonexponential source) },
\end{aligned}
$$

Assuming that, on average, after some oscillations the fraction $\varphi_{0}^{\prime \prime} / \varphi_{0}^{\prime}$ can be approximated by the frequency of the coherent oscillations $\sim m_{\varphi}$, we readily obtain an exponential behavior for the solution of Eq. (17). We may thus write

$$
\phi_{k}^{(2)} \approx-\frac{2 m_{\varphi}^{2}(1-q / 16) B}{M_{p}^{2} m_{\varphi}^{2}(1-q / 4)} e^{q m_{\varphi} t / 2} .
$$

Let us use the following definition for the constant nonlinearity parameter $f_{\mathrm{NL}}^{\phi}: \quad \phi=\phi^{(1)}+f_{\mathrm{NL}}^{\phi}\left(\phi^{(1)}\right)^{2}$. However, the definition is given in $x$ space and we have performed our calculations in $k$ space. In principle we could transform $\phi_{k}^{(2)}$ back to $x$ space, but then we would need to know it for all $k$ and we have evaluated only the superhorizon mode $k=0$. Instead, we can carry the definition of $f_{\mathrm{NL}}^{\phi}$ over to $k$ space by $\phi_{k}^{(2)}=f_{\mathrm{NL}}^{\phi} \frac{1}{(2 \pi)^{3}} \times$ $\int d^{3} k^{\prime} \phi_{k^{\prime}}^{(1)} \phi_{k-k^{\prime}}^{(1)}$ where we have treated $f_{\mathrm{NL}}^{\phi}$ as a constant.

In the present scenario, where the first order perturbations are equivalent to that of a single-field case, we have $\delta^{(1)} \sigma \sim \delta^{(1)} \varphi$ right after inflation. Since, during inflation and preheating $\phi^{(1)}$ stays roughly constant, we immediately obtain an order of magnitude estimate from Eq. (10),

$$
f_{\mathrm{NL}}^{\phi} \sim \frac{\phi_{k}^{(2)}}{\left(\phi^{(1)} * \phi^{(1)}\right)_{k}} \approx-8 \frac{1-q / 16}{1-q / 4}\left(\frac{M_{p}}{\varphi_{0}}\right)^{2} e^{N q / 2},
$$

where we have written $N=t \omega$, where $N$ is the number of oscillations during preheating and $\omega$ is the frequency of the oscillations. On average the frequency of the oscillations is given by $\omega \sim m_{\varphi}$. Inflation ends when $\varphi_{0} \sim M_{p}$, therefore the coefficient in front of the exponential is order one. The factor $B$ in the coefficient of Eq. (18), whose origin lies in the source terms [see Eqs. (14)-(16)], cancels out completely the contribution coming from $\left(\phi^{(1)} * \phi^{(1)}\right)_{k}$, because the initial evolutions for $\delta^{(1)} \varphi$ and $\delta^{(1)} \sigma$ are the same. The above expression should be compared with the observationally constrained one: $f_{\mathrm{NL}}=-f_{\mathrm{NL}}^{\phi}+(11 / 6)$.

The amplitude of the oscillations remains constant in our case. Therefore $N$ is an indicative number, which is valid until the backreaction kicks in and shuts off the parametric resonance. Obviously $N$ depends on a particular potential and also on the expansion of the Universe. The parametric resonance would shift with the expansion as $q \sim \Phi^{2} t^{-2}$ and thus becomes narrower. However, for a simple singlefield chaotic type inflation model with $V \sim\left(m_{\varphi}^{2} / 2\right) \varphi^{2}$, one would typically have many oscillations within one Hubble time: $\omega H^{-1} \sim M_{p} / \Phi \gg 1$. It seems therefore that backreaction would be more decisive as far as the magnitude of the non-Gaussian amplitude $f_{\mathrm{NL}}^{\phi}$ is concerned. This re- 
quires more study, but let us point out that in chaotic inflation backreaction becomes important after 10-30 oscillations [3]. Hence $N=\mathcal{O}(10)$ might be a reasonable number and, should we for illustrative purposes choose $q=0.8$, we would obtain $f_{\mathrm{NL}} \approx e^{4} \approx 55$. This should certainly be at an observable level for the Planck Surveyor mission.

The expansion of the Universe changes the situation in two ways: First, the parametric resonance can be broad with $q>1$ a time dependent quantity [3], and, second, because of the expansion the momenta and the oscillation amplitude redshift. However, the amplitude of the first order metric perturbation still undergoes resonant amplification as the momentum modes drift through the broad resonance regime [5]. This ensures that the second order metric perturbations also grow exponentially, but one has to ensure that the amplitude of the initial perturbations for $\sigma$ does not damp away during inflation. A detailed study would require numerical simulation, but nevertheless we may conclude that our result hints at the possibility of exciting the second order metric perturbations during the first few oscillations of the inflaton, hence linking preheating with possibly observable non-Gaussianities.

It is our pleasure to thank Andrew Liddle for discussions. K. E. is supported partly by the Academy of Finland grant no. 75065. A. V. is supported by the Magnus Ehrnrooth Foundation. A. V. thanks NORDITA and NBI for their kind hospitality during the course of this work.

[1] J. H. Traschen and R. H. Brandenberger, Phys. Rev. D 42, 2491 (1990).

[2] L. Kofman, A. D. Linde, and A. A. Starobinsky, Phys. Rev. Lett. 76, 1011 (1996); Y. Shtanov, J. H. Traschen, and R.H. Brandenberger, Phys. Rev. D 51, 5438 (1995); D. Boyanovsky, H.J. de Vega, and R. Holman hep-ph/ 9701304, and references therein.

[3] L. Kofman, A. D. Linde, and A. A. Starobinsky, Phys. Rev. D 56, 3258 (1997).

[4] D. Cormier, K. Heitmann, and A. Mazumdar, Phys. Rev. D 65, 083521 (2002).

[5] B. A. Bassett, D. I. Kaiser, and R. Maartens, Phys. Lett. B 455, 84 (1999); B. A. Bassett, F. Tamburini, D. I. Kaiser, and R. Maartens, Nucl. Phys. B561, 188 (1999); K. Jedamzik and G. Sigl, Phys. Rev. D 61, 023519 (2000); F. Finelli and R.H. Brandenberger, Phys. Rev. Lett. 82, 1362 (1999); F. Finelli and R. H. Brandenberger, Phys. Rev. D 62, 083502 (2000).

[6] A. R. Liddle, D. H. Lyth, K. A. Malik, and D. Wands, Phys. Rev. D 61, 103509 (2000).

[7] V.F. Mukhanov, H. A. Feldman, and R. H. Brandenberger, Phys. Rep. 215, 203 (1992).
[8] M. Parry and R. Easther, Phys. Rev. D 59, 061301 (1999); R. Easther and M. Parry, Phys. Rev. D 62, 103503 (2000); F. Finelli and S. Khlebnikov, Phys. Lett. B 504, 309 (2001); F. Finelli and S. Khlebnikov, Phys. Rev. D 65, 043505 (2002).

[9] J. McDonald, Phys. Rev. D 66, 043525 (2002); K. Enqvist, S. Kasuya, and A. Mazumdar, Phys. Rev. Lett. 89, 091301 (2002); K. Enqvist, S. Kasuya, and A. Mazumdar, Phys. Rev. D 66, 043505 (2002).

[10] C. L. Bennett et al., Astrophys. J. Suppl. Ser. 148, 1 (2003).

[11] J. Maldacena, J. High Energy Phys. 05, 013 (2003).

[12] J. Lesgourgues, D. Polarski, and A. A. Starobinsky, Nucl. Phys. B497, 479 (1997); J. Martin, A. Riazuelo, and M. Sakellariadou, Phys. Rev. D 61, 083518 (2000); A. Gangui, J. Martin, and M. Sakellariadou, Phys. Rev. D 66, 083502 (2002).

[13] E. Komatsu, astro-ph/0206039; D. S. Salopek and J. R. Bond, Phys. Rev. D 42, 3936 (1990); D. S. Salopek and J. R. Bond, Phys. Rev. D 43, 1005 (1991); A. Gangui, F. Lucchin, S. Matarrese, and S. Mollerach, Astrophys. J. 430, 447 (1994); M. Bruni, S. Matarrese, S. Mollerach, and S. Sonego, Classical Quantum Gravity 14, 2585 (1997); A. D. Linde and V. Mukhanov, Phys. Rev. D 56, R535 (1997); S. Matarrese, S. Mollerach, and M. Bruni, Phys. Rev. D 58, 043504 (1998); N. Bartolo, S. Matarrese, and A. Riotto, Phys. Rev. D 65, 103505 (2002); G. Rigopoulos, Classical Quantum Gravity 21, 1737 (2004); N. Bartolo, S. Matarrese, and A. Riotto, J. Cosmol. Astropart. Phys. 0401, 003 (2004); M. Zaldarriaga, Phys. Rev. D 69, 043508 (2004); V. Acquaviva, N. Bartolo, S. Matarrese, and A. Riotto, Nucl. Phys. B667, 119 (2003); P. Creminelli, J. Cosmol. Astropart. Phys. 0310, 003 (2003); H. Noh and J. Hwang, Phys. Rev. D 69, 104011 (2004); K. A. Malik and D. Wands, Classical Quantum Gravity 21, L65 (2004); I. Rigopoulos, E.P.S. Shellard, and B. J.W. van Tent, astro-ph/0410486.

[14] F. Bernardeau and J. P. Uzan, Phys. Rev. D 66, 103506 (2002); F. Bernardeau and J.P. Uzan, Phys. Rev. D 67, 121301 (2003); I. Yi and E. T. Vishniac, Astrophys. J. Suppl. Ser. 86, 333 (1993).

[15] K. Enqvist and A. Vaihkonen, J. Cosmol. Astropart. Phys. 0409, 006 (2004).

[16] N. Bartolo, E. Komatsu, S. Matarrese, and A. Riotto, Phys. Rep. 402, 103 (2004).

[17] N. Bartolo, S. Matarrese, and A. Riotto, J. High Energy Phys. 04, 006 (2004).

[18] K. Enqvist, S. Kasuya, and A. Mazumdar, Phys. Rev. Lett. 90, 091302 (2003); K. Enqvist, A. Jokinen, S. Kasuya, and A. Mazumdar, Phys. Rev. D 68, 103507 (2003); K. Enqvist, S. Kasuya, and A. Mazumdar, Phys. Rev. Lett. 93, 061301 (2004); K. Enqvist, A. Mazumdar, and A. Perez-Lorenzana, Phys. Rev. D 70, 103508 (2004).

[19] A. R. Liddle and A. Mazumdar, Phys. Rev. D 61, 123507 (2000). 